Extrême-Orient Extrême-Occident

\section{Extrême-Orient Extrême-Occident}

39 | 2015

Corps souffrants dans les littératures de la Chine et du Japon au XXe siècle

\title{
Corps sensible et corps pratico-inerte : femme frustrée et kamikaze mutique dans une nouvelle de Kôno Taeko, «Tetsu no uo » (« Poisson de fer »)
}

Sensitive Body and Practico-Inert Body: Frustrated Woman and Mute Suicide Bomber in Kono Taeko's 'Tetsu no uo' ('Iron Fish')

$$
\begin{gathered}
\text { シェラール・シアリ。踓動する身体と無反応な身体：「鉄の魚」 } \\
\text { (河野多恵子) 一欲求を阻止された女性と沈黙する力ミカゼ。 }
\end{gathered}
$$

\section{Gérard Siary}

\section{OpenEdition \\ Journals}

\section{Édition électronique}

URL : http://journals.openedition.org/extremeorient/538

DOI : 10.4000/extremeorient.538

ISBN : 978-2-84-292-449-2

ISSN : 2108-7105

Éditeur

Presses universitaires de Vincennes

Édition imprimée

Date de publication : 1 octobre 2015

Pagination : $177-202$

ISBN : 978--84292-447-8

ISSN : 0754-5010

Référence électronique

Gérard Siary, « Corps sensible et corps pratico-inerte : femme frustrée et kamikaze mutique dans une nouvelle de Kôno Taeko, «Tetsu no uo » («Poisson de fer ») », Extrême-Orient Extrême-Occident [En ligne], 39 | 2015, mis en ligne le 01 octobre 2017, consulté le 01 mai 2019. URL : http:// journals.openedition.org/extremeorient/538; DOI : 10.4000/extremeorient.538 


\title{
Corps sensible et corps pratico-inerte : femme frustrée et kamikaze mutique dans une nouvelle de Kôno Taeko, «Tetsu no uo » («Poisson de fer »)
}

\author{
Gérard Siary
}

\begin{abstract}
« Notre chair n'est rien d'autre que cela qui s'éprouvant, se souffrant, se subissant et se supportant soimême et ainsi jouissant de soi selon des impressions toujours renaissantes, se trouve, pour cette raison, toujours susceptible de sentir le corps qui lui est extérieur, de le toucher aussi bien que d'être touché par lui. Cela donc dont le corps extérieur, le corps inerte de l'univers, est par principe incapable ${ }^{1}$.»
\end{abstract}

S'il est une expérience dont pâtit le corps entre émotion et sensation, au mental et au physique, c'est bien celle de la guerre. Même si elle s'étale sur la longue durée, l'individu cherche à lui opposer tant bien que mal une résistance, et même une résilience, pour se forger une identité viable. Il s'ensuit une sorte de dialectique du mal-être et de la guérison, de la dépendance et de la libération. $\mathrm{Du}$ mal-être au sens large, d'un moment physiquement ou moralement douloureux qu'on ne parvient pas à dépasser, à surmonter, à reléguer dans un passé révolu ou à intégrer dans sa trajectoire existentielle. De la dépendance, c'est-à-dire de la fixation sur une phase de vie qu' on peine à comprendre et qui pèse sur le présent si l'on n'y porte remède.

Ce rapport privilégié que le corps souffrant tisse avec la guerre, privilégié car celle-ci bouleverse le centre de gravité de tout le corps social au risque d'écarteler le sujet entre la nation et la famille, la littérature peut en rendre compte à sa façon, qui ne s'accorde pas forcément avec celle de la science historique. Elle peut dénoter le vécu de l'individu acteur, sur le moment ou presque, ou de l'individu témoin, à distance de l'événement, ainsi que le fait l'Histoire, mais elle recourt aussi à d'autres formes d'expression, en particulier la métaphore, qui peuvent procurer, dans une forme-sens, une autre vision du

1. Henry $2000: 8-9$. 
monde que celle de l'Histoire et peut-être réconcilier l'être humain et son milieu.

\section{L'expérience de la guerre à travers le corps féminin et dans la littérature féminine}

Le corps souffrant dont il est question ici s'écrit dans une nouvelle en japonais qui concerne le traumatisme de la guerre perçu d'un point de vue féminin. Datée de 1976, d'une époque où le syndrome post-traumatique est peu usité, elle s'intitule « Tetsu no uo », « Poisson de fer ». La narratrice y conte que l'amie qu'elle accompagne dans un musée où l'on vénère les soldats morts à la guerre, s'y fait enfermer la nuit. Là, elle se niche dans la nef sous-marine, dite le poisson, où son défunt soldat de mari, durant une guerre innommée, a trouvé la mort en percutant une autre nef, dite poisson géant. À sa façon, elle y revit le lien qu'elle n'a jamais eu avec un époux trop vite disparu et revivifie son propre rapport au monde de femme mariée. Cette trame sera analysée plus loin, mais le poisson de fer apparaît d'emblée comme un espace létal, un corps qui cristallise le vécu douloureux de la guerre, tant pour le mari que pour la veuve, et le corps national, mais aussi, pour cette femme, paradoxalement, un lieu où renaître, où se refaire, où se comprendre enfin.

L'auteur est une femme de lettres du Japon, Kôno Taeko (1926-2015), qui a vécu la guerre d'Asie-Pacifique (1937-1945), souvent plus à l'usine qu'à l'école, et dont la production passe pour scruter la condition féminine, alors sous-traitée dans la société à domination masculine du Japon, tester des voies de salut inédites, notamment dans les pratiques sexuelles (échangisme, pédophobie, sado-masochisme, etc.) et expérimenter des formules littéraires relativement incongrues. Au début de sa carrière, elle compose plutôt à partir de son propre vécu de la guerre d'Asie-Pacifique. Nombre de ses récits sont d'ailleurs repris dans les anthologies de la fiction sur ces années de guerre. Mais, dans les années 1970, elle amorce un nouveau thème, celui de la liberté, pour la femme en particulier.

À cet égard, «Poisson de fer » conjugue au mode féminin l'expérience singulière et collective de la guerre, la quête de la liberté par voie transgressive et l'approche métaphorique de l'objet, (le poisson) quasi anhistorique, qui lui confère valeur morale. Tout cela passe par une phénoménologie du corps et même de la chair qui souffre à la vue ou à l'idée de la souffrance du corps d'autrui, qui souffre aussi de la frustration qu'engendre le deuil, qui souffre enfin sous la chape de l'histoire officielle imposée, mais qui, au fil du temps, résiste. Et c'est justement ce corps du poisson de fer qui concentre cette souffrance et la communique au lecteur. 
Corps sensible et corps pratico-inerte : femme frustrée et kamikaze mutique

\section{Corps exproprié/Corps approprié}

Le(s) sens du corps souffrant ne peu(ven)t se déterminer à partir d'une définition préalable dudit corps. Tout dépend de sa place et de sa fonction dans la structuration d'un écrit donné. C'est après, dans un second temps, que le(s) sens dégagé(s) peuvent être ramenés à des formes ou à des concepts qui les subsument - mais pas nécessairement, car la littérature peut aussi entrouvrir des pistes ignorées de l'histoire, de la philosophie, de l'anthropologie. Aussi la lecture serrée de la nouvelle de Kôno Taeko dans la perspective du corps souffrant passe-t-elle d'abord par l'analyse de la diégèse, qui relate un lent cheminement de l'expropriation du corps du mari à l'appropriation de ce même corps via le corps du poisson de fer, et à travers laquelle émerge un corps souffrant singulier. Comme il n'existe pas de traduction française de ce texte assez peu connu et que ses plus petits détails s'imbriquent étroitement dans son économie narrative et symbolique, le fil du texte sera suivi au plus près. La paraphrase qui suit l'épouse parfois jusqu'à traduire littéralement certains passages. Ainsi le lecteur ressentira-t-il au plus près ce corps en souffrance, qui se manifeste autant par le dit que par le non-dit. Le commentaire qui suit renvoie entre parenthèses aux paragraphes concernés du résumé. La numérotation des paragraphes n'est pas celle de l'original, mais celle de l'auteur de l'article.

\section{La disparition de l'amie et la mort étrange des jeunes filles}

$\S 1$ - Le narrateur unique, femme anonyme, ouvre le récit en première personne sur le «nous » qu'elle forme avec son amie au moment où elles s'apprêtent à sortir du musée, non nommé, dont la gardienne annonce la fermeture des portes, un peu trop tôt pour certains visiteurs. C'est alors que l'amie, anonyme aussi, s'excuse auprès d'elle, lui demande de l'attendre un instant, lui confie son parapluie car le temps s'est remis au beau et retourne à l'intérieur du musée. L'amie sort sans se retourner, se disant qu'il était inutile de lui offrir de prendre son parapluie. En effet, selon elle, la vraie raison qui fait qu'elles se sont si longtemps attardées devant les pièces exposées au rez-de-chaussée, c'est que sa compagne n'avait nulle envie de rentrer, rien que de raisonnable. De plus, le passage dans le hall avait dû lui inspirer quelque regret. Elle croit saisir que sa compagne ne songeait qu'à réintégrer le musée pour laisser libre cours à ses émotions avant de ressortir avec un mouchoir à la main.

$\S 2$ - Puis la narratrice s'engage sur un sentier jonché de feuilles d'automne, à distance du bâtiment du musée qu'elle aperçoit désormais de loin et qu'indique un panneau en forme de flèche. Elle foule au pied les feuilles mortes, qu'elle remue des deux parapluies, et se rappelle ces pièces exposées en vitrine au musée, laissées par 
des hommes en guise de testament, qui contiennent des feuilles et des fleurs, pâlies avec le temps, dont certaines ont été soigneusement et laborieusement découpées et collées une à une par des jeunes filles figurées en photo dans la même vitrine, toutes mortes d'une mort étrange car causée par un grand feu déclenché à distance. Et la narratrice de se dire qu'elle aussi, à une autre époque, a accompli les mêmes gestes pour des compositions florales, qui ont toutes disparu dans un grand incendie enclenché au loin par un certain mécanisme, mais qu'à la différence des jeunes filles elle a pu échapper à cette mort étrange.

$\S 3$ - La narratrice attend en vain que son amie revienne après avoir essuyé ses larmes, avec un mouchoir. Comme toutes les portes du musée se referment, elle glisse par la serrure un message à la disparue qu'elle entend soudain lui dire, de l'autre côté de la porte, de rentrer chez elle sans s'inquiéter, et qu'elle lui expliquera à l'occasion ce qui s'est passé. La narratrice tremble de tout son corps. Elle éprouve comme un vertige. Il lui semble entendre les mots d'un moine qui va s'enterrer vivant, et voir tournoyer au-dessus de sa tête le contenu des vitrines, les sabres, les taches de sang roussies avec le temps et les idéogrammes des innombrables lettres d'adieu. Dans la nuit, trois images la poursuivent : son amie se retrouve dans la situation du moine de la légende du temple Dôjôji, qu'un démon féminin a enfermé sous cloche, et la narratrice est alors en proie à une gastralgie ; son amie a voulu commettre quelque acte criminel, mais la narratrice n'y croit pas et se déclare même solidaire des actes de celle-ci ; son amie a été victime d'une destinée néfaste. Quatre ans après, son amie s'ouvre à la narratrice de ce qui s'est passé cette nuit-là, et celleci rapporte les faits comme elle se les imagine. Le récit passe de la première à la troisième personne, l'amie devient protagoniste à part entière.

\section{La mort étrange du premier mari et l'ajournement de la visite au musée}

$\S 4-\grave{A}$ un moment et en un lieu, à l'insu de son épouse elle-même, le premier mari de la protagoniste avait fait ses adieux à ses camarades pour monter dans le ventre d'un poisson de fer et filer au large sous les yeux de ceux qui restaient en arrière. Très loin de là, il avait piqué sur le ventre d'un poisson de fer géant à demi émergé, telle une île en fer, et trouvé la mort dans l'explosion du corps de son propre poisson. Sa chair, éparpillée en mille morceaux au fond de la mer, avait sûrement vite attiré de vrais poissons. Comme lui aussi était mort d'une mort sans doute étrange, et même s'il y avait des différences de degré dans l'étrangeté, il avait été déifié, avait-elle entendu dire, ainsi que maints congénères masculins et juste quelques femmes, tous également morts d'une mort étrange. 
$\S 5$ - Elle n'avait jamais visité le lieu où son mari avait été déifié. Elle n'était pas mariée depuis un an que son mari avait pris congé d'elle pour ne revenir que deux fois au foyer et en repartir aussitôt, sans jamais dire ni d'où il venait ni où il retournait. Puis, cent soixante-deux jours après l'ultime visite de son mari, on l'avait été avisée qu'il n'était plus et que la chose était advenue depuis cent vingtdeux jours. Alors même qu'elle était bien au courant de cette déification, elle ne s'était pas empressée de visiter l'endroit où son mari était ainsi déifié. Moins par doute sur le sens de cette déification que par son peu d'envie de rendre en un lieu qu'elle imaginait plongé dans l'ombre. Elle irait un jour, s'était-elle contentée de répondre aux interpellations d'autrui à ce sujet, tout en pensant le contraire, à savoir qu'elle s'en fichait, pour user d'une expression qu'elle n'avait jamais utilisée.

$\S 6$ - Mais plus que l'obscurité du lieu, la vraie raison était que le temps n'était pas encore venu pour cela. Elle voulait sentir son défunt mari complètement à elle, mais n’y parvenait pas. Encore des années après, même après que l'opinion publique eut cessé de réagir, elle n'était toujours pas parvenue à sentir personnellement son mari. Avec le temps, bien qu'elle eût fini par s'approprier ce mari, elle avait perçu ce lieu comme encore plus obscur. Elle se disait à présent qu'il ne valait pas la peine d'y aller. Elle trouverait ledit lieu de plus en plus obscur, mais finirait bien par aller un jour vérifier sur place ce qu'il en était. Néanmoins, même si elle visitait ce lieu, elle ne pensait pas pouvoir se l'approprier à sa guise et à loisir et, quand bien même c'eût été le cas, à remettre de l'ordre dans tout cela.

$\S 7$ - Entre-temps, sept ans après la mort de son premier mari, à l'âge de vingtsept ans, elle s'était remariée, et les années de son second mariage avaient filé aussi vite que celles du premier. Durant toutes ces années, elle n'avait pas non plus visité le lieu, non par respect envers son second mari, mais parce qu'elle était sûre qu'elle irait vérifier un jour ce qu'il en était de l'obscurité du lieu. Elle était aussi rassurée de savoir qu'elle ne s'était pas rendue là-bas par respect pour son second mari au risque de négliger le premier, et qu'elle n'avait pas non plus été là-bas par respect pour son premier mari au risque de négliger le second. Elle s'était sentie d'autant plus naturellement rassurée qu'elle avait vécu loin dudit lieu après son second mariage. Et c'est subitement, lors d'un voyage, qu'elle avait décidé d'aller à l'endroit où son mari était déifié, et demandé à la narratrice de l'accompagner - vingt-cinq ans après. 


\section{Le poisson, objet muséal : de l'information à l'émotion}

$\S 8$ - À son arrivée, par un jour d'automne pluvieux, elle avait trouvé un endroit clair et lumineux. De larges voies, une allée bordée d'arbres géants, des surfaces vertes comme dans un parc, une place couverte de gravillon et ornée d'arbustes. Si cet endroit avait été aussi sombre qu'elle se l'imaginait, elle aurait commis une double négligence : vis-à-vis du premier mari, pour n’y être pas allée de si longtemps, ainsi que du second mari, pour y être allée quand même. Si tel avait été le cas, elle aurait même regretté d'être venue, mais elle pouvait à présent penser comme elle le voulait.

§ 9-Le bâtiment où son mari était déifié de concert avec un grand nombre d'hommes et quelques femmes, était fait de pierre naturelle, d'énormes pièces de bois naturel et de métal. Son mari aurait sûrement voulu se retrouver en bien d'autres lieux. Il n'avait peut-être pas vu celui-ci de son vivant, mais il devait avoir l'intention d'y être déifié en montant dans le poisson de fer. Vu la clarté du lieu, il ne l'aurait pas ignoré et pourrait bien s'y manifester à titre exceptionnel. La décoration extérieure faisait largement honneur au lieu. Des petits écriteaux pendaient aux arbres. L'un d'eux invitait les esprits des morts. Son mari pourrait bien venir sans y être invité, s'était-elle dit. Un autre panneau invitait les esprits à reposer en paix. Elle avait alors pensé que son premier mari connaissait tant de gens qui étaient encore en vie, elle comprise, qu'il ne songeait pas à reposer en paix de sitôt.

$\S 10$ - Après s'être laissé enfermer dans l'un des bâtiments annexes et avoir coupé court aux appels extérieurs, elle était d'abord allée toucher le poisson de fer, poser dessus ses deux mains, caresser cette peau rêche et rouillée comme celle d'un poisson caressé à rebrousse-écailles. Puis, elle était passée par-dessous la barrière en bois. S'étant rapprochée, elle avait couché son corps sur le poisson et, à défaut de pouvoir l'embrasser, elle avait fait le geste de l'enlacer, car elle sentait de tout son corps que c'était son droit et son devoir. Plus d'une centaine de poissons semblables avaient ainsi coulé corps et biens au fond de la mer, mais l'un d'entre eux, on ne savait lequel, avait été retrouvé avec un ventre en partie intact, remonté à la surface et installé là.

$\S 11$ - En apercevant ce ventre allongé et rond au milieu de la salle, elle n'avait pas saisi ce que c'était. Grâce aux panneaux d'explication, elle avait compris que son mari était monté dans ce poisson de quatorze mètres de long mais d'un mètre de diamètre, puis localisé l'orifice par où il était entré. En voyant alors de ses yeux le réceptacle vide et obscur, de moins d'un mètre, où était entré son époux, elle n'avait pu dire avec des mots que son premier mari s'était enfermé lui-même là-dedans 
Corps sensible et corps pratico-inerte : femme frustrée et kamikaze mutique

pour ne pas en ressortir, et à quel point il avait dû étouffer. Quand la porte s'était refermée, il avait dû ressentir une sensation d'étouffement.

$\S 12$ - Elle n'y avait plus repensé un instant, mais ce n'était pas parce qu'elle avait vu à l'étage des pièces qui lui avaient remué le cœur. De celles que lui avait laissées son mari en partant, elle en avait donné à deux ou trois personnes, pour ne garder qu'une décoration et quelques photos enfouies dans un sac. Pour elle, les pièces exposées ne valaient pas la peine de l'être.

\section{Le poisson de fer, corps à vivre de l'intérieur, entre Éros et Thanatos}

$\S 13$ - En voyant l'attitude de son accompagnatrice qui, s'écartant brusquement de la vitrine des écrits autographes et ponctués des mots : « Je n'ai pas de dette, je n'ai pas de liaison avec une femme, je n'ai pas commis de crime », lui avait tourné le dos pour essuyer quelques larmes en vitesse, elle s'était dit que certaines personnes, qui avaient vécu cette époque-là mais n'avaient pas vécu cette expérience au plus profond d'eux-mêmes, ne pouvaient au contraire s'empêcher d'avoir des réactions sensibles. Face à la ferveur de son accompagnatrice, elle avait regardé plus à loisir les pièces exposées.

$\S 14$ - Mais elle avait fait demi-tour vers le poisson et repensé à son étouffante exiguiité. Cette sensation d'enfermement s'était accrue quand l'une des portes du musée s'était refermée pour plonger le lieu dans une semi-obscurité. Elle avait attendu que le bâtiment se fût vidé entièrement et que les portes fussent verrouillées pour enfin éprouver l'impression que cela faisait de se trouver enfermée dans le poisson. Elle avait l'impression que la porte allait comme enfermer son premier mari dans ce ventre étouffant, qu'elle pourrait en faire de même et que c'était déjà presque fait.

$\S 15$ - Malgré l'extinction des feux, une légère clarté filtrait encore, sans doute d'une fenêtre de la cage d'escalier. Le poisson de fer semblait immergé dans les profondeurs, mais le fond de la mer dégageait un peu de lumière, due au reflet des petits cailloux blancs. Elle avait caressé la blancheur dure, granuleuse du fond de la mer, et saisi à pleines mains les cailloux, comme l'avaient fait les enfants. Elle avait alors songé que si la chair de son premier mari, quand elle s'était dispersée au fond de la mer, n'avait pas servi de pâture aux poissons, c'est qu'elle s'était fracassée et disséminée parmi les cailloux. 
$\S 16$ - Son mari, bien que jeune, était d'humeur difficile, peut-être du fait qu'ellemême était jeune, et puis aussi parce qu'il était immature et maladroit, tout comme elle, et qu'elle n'avait pas songé à détendre l'atmosphère. Elle ne se rappelait de lui que son expression favorite, «On doit faire vite », justifiée par l'époque, mais sans doute aspirait-il à former plus franchement un couple avec elle. Il voulait parfois signifier par là qu'ils étaient très jeunes mais plus seuls du tout et devaient faire tous leurs efforts pour qu'on les traite vite comme des adultes. Il avait même utilisé cette expression au lit. Elle avait perçu plus tard la drôlerie du mot. Sur le moment, elle s'était assise pour écouter les admonestations. Et puis son mari avait eu une autre expression : «Je veux que tu fasses... » ceci ou cela, ou bien encore : « Je ne veux pas que tu dises n'importe quoi » parce qu'il pensait qu'ils devaient se presser. «Qu'aurais-tu voulu que je fasse ? », avait-elle dit alors, éclatant d'un rire mêlé de sanglots, et se couvrant la bouche.

$\S 17$ - Puis elle était entrée en se baissant dans le ventre du poisson, avait touché les arceaux à droite et à gauche, tenté en vain de se redresser, s'était baissée davantage jusqu'à se courber et avait gardé cette position un moment. Ensuite, elle s'était accroupie pour caresser le bas du poisson en disant : «N'as-tu pas pensé à ce que tu aurais voulu que je fasse à cette époque-là ? » Ses mains avaient touché la chair fine, dure et épineuse du poisson. Son mari n'avait-il pas alors désiré revoir la lumière du soleil ? Pas de réponse. Rien n'avait changé entre son mari qui ne réagissait pas, et elle, qui ne savait comment le faire réagir.

\section{Le poisson au prisme du temps : de l'obscurité à la clarté solaire}

$\S 18$ - Comme son premier mariage avait été fort bref, le second avait fini par durer beaucoup plus de temps. De toutes ces années, quand il lui était arrivé de repenser à son premier mari, la part de sa vie de femme qu'elle lui avait consacrée lui avait paru d'une violente injustice en comparaison de son second mari, et ce fut de son fait. Et puis, cela ne lui avait plus semblé si étrange. L'obscurité avait encore gagné. Elle avait trouvé à tâtons une chaise pliante dans la salle de conférences, et l'avait posée devant la barrière en bois. Même sept ans après s'être remariée, il lui semblait l'avoir fait trop tôt, mais cela tenait à ce que son second mari avait deux ans de plus qu'elle et que cette pensée la faisait se sentir libre vis-à-vis de lui.

$\S 19$ - Lorsqu'elle en était venue à parler par hasard de son premier mari au second, celui-ci avait désigné celui-là comme «l'homme en partance pour la guerre ». Elle avait vu alors le caractère pour la guerre, jadis courant, grossir sous ses yeux, et à ces mots, «l'homme en partance pour la guerre », elle avait cru revoir l'image de 
Corps sensible et corps pratico-inerte : femme frustrée et kamikaze mutique

ce premier mari avec une intensité redoublée. Son second mari, peu sensible à sa situation, lui avait aussi dit qu'elle avait dû faire du bien dans une vie antérieure pour qu'un homme aussi gentil que lui veuille la reprendre - encore qu'il ne sût pas trop... -, et par cette plaisanterie, signifié qu'elle ne devait pas prendre les choses trop à cœur. Ces mots, peut-être trompeurs, l'avaient attachée jusqu'à ce jour à son second mari.

$\S 20$ - Il lui était arrivé de se demander si son premier mari, en s'enfermant dans le ventre du poisson de fer, avait eu des désillusions et des regrets à son endroit, mais alors, les regrets et les désillusions d'un homme difficile. Et de penser qu'elle aurait pu faire un petit peu plus pour lui au cours de leur vie conjugale. Accoudée dans le noir sur le rebord de la barrière, elle s'était dit que ces pensées auxquelles elle en était arrivée tenaient à sa seconde vie conjugale avec son second mari, et puis que ce qu'elle avait vécu avec le premier lui avait fait éprouver un profond bien-être en entendant les mots de son second mari au tout début de leur mariage. Si quelqu'un lui avait fait entendre cela, elle le saurait si elle revoyait encore une fois la lumière resplendissante du soleil.

Toute la nouvelle tourne autour de la difficulté du lien à restaurer avec le mari, de ce rapport à instaurer puisqu'ils n'ont jamais vraiment communiqué ni sans doute eu de commerce sexuel. Et cela débute dès lors que la gardienne du musée - le personnel du récit est largement féminin - annonce sa fermeture immédiate. Devoir sortir, c'est se plier à la temporalité muséale qui véhicule la représentation de l'Histoire selon un mode d'expression imposé, qui la médiatise par des vitrines. Sortir du musée, pour la protagoniste, c'est se faire exproprier du corps de son premier mari, ne pouvoir nouer le lien qu'elle est précisément venue établir. S'approprier son premier mari, tel est bien le «thème en puissance » de ce récit. Pour cela, il faut contourner cet appareil institutionnel qui éloigne les corps les uns des autres. Le mort est ici doublement éloigné : il a disparu corps (et biens) ; il n'est plus qu'objet in absentia de la représentation historique et religieuse que le musée impose plus qu'il ne propose, il n'appartient plus, en l'occurrence, à son épouse qui doit passer par des voies indirectes afin de le retrouver, et à toutes les épouses en général.

Et tel est bien aussi le «thème en effet » de ce récit : elle se réapproprie son mari ou du moins comprend-elle assez pourquoi elle n'a pu se l'approprier à l'époque pour qu'elle puisse passer de l'obscurité à la lumière du soleil et revivre. Entre ces deux pôles, l'expropriation et l'appropriation, le récit se déploie par des dégagements successifs qui se jouent dans le rapport que la femme, ou plutôt les femmes, la narratrice et sa protagoniste, tissent au fil du 
temps avec l'histoire collective (la mort étrange des jeunes filles), individuelle ensuite (la mort étrange du premier mari), et enfin conjugale (le tissu conjonctif de l'interaction maritale). Mais c'est la figure de ce poisson-mari et peut-être plus que cela, dont la protagoniste ne veut pas s'éloigner, au sein duquel elle pénètre comme dans un espace masculin interdit et d'où elle veut ressortir, qui relie toute cette trame.

\section{Médiatisation de la protagoniste et de l'Histoire}

Avant d'explorer ces pistes, il importe d'exposer trois difficultés du récit. La première tient à ce que dans cette nouvelle à récit enchâssé, l'expérience de la veuve n'est pas rapportée par elle, par exemple au style direct, mais par l'accompagnatrice, de façon transposée. La seconde, à ce que le référent historique y est soigneusement gommé. La troisième, à ce qu'en lieu et place de l'Histoire apparaît une légende actualisée, avec ambiance insolite, sinon fantastique. Dans les trois cas, il y a une prise de distance modulée, qui reste à interpréter et qui peut l'être en fonction du corps souffrant.

\section{Un point de vue distancié}

Dans la distribution des rôles, entre narratrice et protagoniste, le positionnement dans l'espace-temps est remarquable. Après leur visite commune du musée, alors que la narratrice s'éloigne du musée avant de méditer ce qu'elle y a vu, de l'extérieur vers l'intérieur, la protagoniste s'y enferme et va s'engouffrer dans le poisson pour ensuite l'observer à distance, de l'intérieur vers l'extérieur. On s'approche et on s'éloigne par degrés de l'objet central, le poisson, ce qui atteste le souci de se saisir de l'objet, puis de prendre une certaine distance vis-à-vis de lui.

En alternant la progression vers le musée-poisson et les étapes de la vie de femme mariée de la protagoniste, le récit enchâssé remonte du lointain passé, avec le départ du premier mari, au présent de l'enfermement volontaire dans le musée, en passant par l'expérience bienfaisante du second mariage. De façon circulaire, le moment où la protagoniste s'apprête à remonter précède alors celui où la narratrice reçoit le récit qu'elle va restituer, et la boucle est bouclée : il est temps de sortir du musée et du récit.

Celui-ci s'ordonne en deux parties nettes : les deux femmes se séparent au musée, puis l'une rapporte la nuit passée dans ledit musée par l'autre. Cependant il est tout entier rapporté du point de vue de la narratrice, l'accompagnatrice, qui se pose en conteuse. Que le récit enchâssé ne soit pas du tout assumé par 
la protagoniste a de quoi étonner le lecteur même habitué à pareilles histoires d'étranges incidents. Mais la narratrice, qui prend elle-même ses distances avec l'objet, le musée, le contenant, représente une protagoniste qui prend elle aussi ses distances avec le poisson, le contenu.

$\mathrm{Si}$, dans la première partie, la narratrice est homodiégétique, présente en tant que personnage de l'histoire, dans la seconde, le récit enchâssé, elle est hétérodiégétique, extérieure au récit, et intervient peu, mais il n'en demeure pas moins que tout émane d'elle. En d'autres termes, elle est la porte-parole d'une expérience dont elle a le sentiment que son amie l'a éprouvée, mais qui ne lui a pas été communiquée telle quelle (§3), comme si le vécu de son amie ne pouvait se dire au style direct. C'est parce qu'elle est apte à ressentir l'émotion de son amie que la narratrice s'estime qualifiée pour raconter à sa façon ce qu'elle a entendu. Dotée d'imagination, elle brode déjà sur les mobiles qui amènent son amie à s'enfermer la nuit au musée, imagine déjà les objets qui l'assaillent comme dans un cauchemar (§3), puis nourrit son imaginaire des révélations de son amie. De la sorte, elle passe de la mort étrange des jeunes femmes à la mort tout aussi étrange du premier mari de son amie. Le temps qui s'éclaircit contribue aussi à placer la narratrice en phase avec la protagoniste, qui s'étonne de la clarté de l'atmosphère $(\S 8)$. La peur que celle-ci soit enfermée dans le musée prédispose celle-là à imaginer l'enfermement qu'a connu le mari de son amie. Ces éléments de qualification font de la narratrice comme le double de son amie et, de façon plus large, la porte-parole, à titre collectif et individuel, de toutes ces femmes qui ont pâti de la guerre, dont elle n'a pourtant pas partagé le destin mais qu'elle fait parler, avec ses mots à elle, au style indirect.

Cette régie narrative, qui a sa propre cohérence, se caractérise par le souci structural de prise de distance dans l'espace-temps, relayée par plusieurs instances, avec l'incident au cœur du récit : la mort étrange. Cette distanciation réitérée figure la difficulté d'approcher directement cette étrange mort, et de la dire autrement que par une voie indirecte, qui est ici la métaphore, à savoir le poisson. C'est lui le corps qui, abordé par transgression de la barrière muséale, redonne un semblant d'unité à la vie de l'amie. C'est lui le corps imaginé (un poisson au lieu d'un bateau), encore une expression indirecte, qui renferme la clé de l'énigme et s'inscrit comme objet d'herméneutique.

\section{Un référent historique indirect}

Cette prise de distance, qui médiatise l'expérience du corps souffrant sans l'anesthésier, est aussi notable dans le rapport à l'Histoire. Nombre d'éléments sont identifiables avec précision. Le musée dont il s'agit n'est autre que le 
Yûshûkan, édifice attenant au sanctuaire de Yasukuni, sis à Tokyo, situé au bout d'une large et belle avenue dégagée et bordée d'arbres, lieu édifié pour, comme l'indique le panneau, le repos des âmes des soldats morts à la guerre et devenus des dieux vénérables comme tels. Les lettres exposées sont notamment celles qu'ont laissées les pilotes des unités spéciales d'assaut, dites tokkôtai, plus connues sous le nom de kamikaze, avant de partir pour une mission sans retour, et ce avec la mention précise d'une lettre-testament, et de fleurs séchées, peut-être de ces fleurs de cerisier dont le caractère éphémère évoque le destin coupé net des jeunes pilotes. Le poisson de fer n'est autre qu'un engin de guerre exposé, la torpille sous-marine (kaiten) pilotée par ces unités spéciales dont le mari faisait partie en 1944. Quant au mécanisme enclenché à distance, l'allusion est plus vague, et les grands incendies peuvent renvoyer à la bombe atomique ou aux bombardements américains qui ont marqué la dernière partie du conflit.

Le cas du premier mari est envisagé à la fois dans et hors de l'Histoire. Dans l'Histoire, au sens le plus fort, car il fait partie de ces unités spéciales d'assaut qui, à bord d'une torpille à pilotage manuel lourdement chargée d'explosifs, fonçaient sur un bâtiment ennemi, si possible un porte-avions, pour le faire exploser et exploser avec elle. Les pilotes étaient des jeunes gens dans la fleur de l'âge, entre seize et vingt-cinq ans. La réussite de la mission impliquait la mort du pilote qui menait son engin sur la cible. En reconnaissance de cet acte d'autosacrifice à l'empereur et à la nation, le soldat était promu dieu de la guerre (gunshin) et honoré post mortem au sanctuaire Yasukuni. Cela, c'est l'histoire officielle, celle qu'offre le musée, avec en particulier les lettrestestaments et poèmes d'adieu laissés par les jeunes gens avant leur sortie fatale (voir annexe). Secret défense oblige, les familles n'étaient pas toujours au courant de l'activité de l'intéressé ni du lieu où se trouvait sa base.

Il n'est pas sûr qu'il s'agisse là d'ironie, comme l'insinue la critique, car le propos présuppose du lecteur un savoir qui, en 1976, est peut-être encore mobilisable, ce qui n'est pas sûr. S'il y a bien ironie, alors la nouvelle s'en prend expressément au sanctuaire Yasukuni qui glorifie de façon indirecte les soldats morts et la guerre d'Asie-Pacifique menée par le Japon, ce qui ne va pas sans susciter des remous et controverses politiques ${ }^{2}$. Derrière l'expérience distanciée de nos deux femmes, le traumatisme de la guerre, avec retour du refoulé. Vingt-cinq ans d'oblitération, d'atermoiements et de rationalisation pour que la veuve visite le sanctuaire Yasukuni, traduisent la difficulté à intégrer la violence de l'Histoire - à commencer par la violence du silence.

2. Takahashi 2005. 
Inversement, mais cela n'est pas incompatible avec ce qui précède, ce gommage historique profite à une sorte de fable qui déplace l'accent sur le plan du gender. Il s'agit en effet d'une femme qui, dans un lieu hautement symbolique, mais surtout consacré à une majorité d'hommes, raconte, au milieu de femmes (les gardiennes), une expérience féminine qui combine la sienne propre et celle de son amie, et qui raconte surtout le vécu de la protagoniste. Celle-ci, en essayant de comprendre une mort étrange sur la longue durée, cherche à rétablir une continuité existentielle que, certes, l'Histoire a bouleversée.

\section{Une légende médiane}

Pour autant, le symbole du poisson de fer, au cœur de la nouvelle, invite à s'interroger sur sa présence ici quand il eût été possible d'appeler un chat un chat, et, comme telle, une torpille sous-marine à guidage manuel. La nouvelle, qui présente les traits du genre, porte un titre introduisant une dimension étrange, voire fantastique, qui ne sied pas a priori à ce à quoi renvoie le poisson, l'expérience vécue de la guerre, étalée sur plusieurs années de vie(s) de femme(s). Mais, pour la narratrice, la formulation curieuse de certains détails (mécanisme enclenché à distance, description de l'attitude inquiétante de son amie, sentiment de mort étrange) favorise le passage à une autre ambiance insolite et à la métaphore du poisson.

Le propos actualise à sa façon la légende du temple Dôjôji que la narratrice propose en clé d'explication possible du comportement de son amie ( $\$ 3$ ). C'est un récit bouddhique qui apparaît dans les recueils de setsuwa (anecdotes) comme le Dainihon Hokkekyô genki (Relation des miracles) effectués par le Sutra du Lotus au Japon (ca 1040-1043) et dans le Dôjôji engi emaki, son illustration écrite et visuelle la plus ancienne. Ce rouleau raconte l'histoire d'une jeune aristocrate qui veut séduire un jeune bonze en pèlerinage à Kumano. Pour lui échapper, le bonze promet qu'il repassera, mais n'en fait rien. Folle de passion, la femme se mue en serpent et le poursuit jusqu'au temple Dôjôji. Les autres bonzes cachent le jeune homme sous une cloche à laquelle la femme-serpent (et quelque peu dragon) met le feu. Plus tard, en rêve, le vénérable du temple voit le jeune homme qui lui demande de réciter pour lui le sutra du lotus car la femme serpent l'a violé et il est revenu sur terre sous la forme d'un serpent. Le vénérable sauvera et le bonze et la malheureuse jeune fille.

La cloche, dans la nouvelle, est remplacée par le poisson-mari que va hanter la veuve, l'ex-épouse qu'il n'a pas considérée ( $\S 18$ ), afin de se l'approprier ( $\$ 6)$. Mais les rôles sont passablement inversés. L'homme 
devient l'animal, sans se confondre avec le poisson, qui est tout de même le réceptacle qui l'avale à jamais. La femme va bien le traquer, tout comme dans l'anecdote bouddhique, mais elle est plutôt la victime du mari, lequel pâtit de son enfermement volontaire au sein d'un animal déclencheur de feu, qui lui coûtera la vie, et apparaît aussi en victime. En ce lieu censément voué au repos des esprits masculins, avec juste quelques femmes pour la forme ( $\$ 9)$, s'accomplit une sorte d'exorcisme en vue de sauver l'âme de la protagoniste, mais aussi de permettre la réconciliation entre vivants et morts. Il y a lieu de se demander - la question est même posée expressément à l'issue du récit (§20) - si quelque présence occulte, comme l'esprit dudit premier mari dont la manifestation n'est pas exclue ( $\S 8)$, ne vient pas se manifester de quelque façon pour redonner une ligne de sens à la vie de son ex-épouse. L'idée que les âmes de Yasukuni souffrent aussi affleure. La présence du poisson introduit une dimension spirituelle insolite. La légende fonctionne ici comme un mythe qui, actualisé, cristallise une époque et donne sens à une société face à la violence de l'Histoire.

\section{Le corps souffrant à l'échelle collective}

C'est à l'instant même où la protagoniste, qui vient d'éprouver une certaine émotion, risque d'être éconduite en raison du règlement intérieur du musée, qu'elle décide de transgresser ( $\$ 1$ ). Cette rupture avec l'ordre des choses résulte de l'interaction entre les corps meurtris de ceux qui ont péri à la guerre et les corps des spectateurs sensibles à leurs souffrances.

Lorsque les visiteuses se voient invitées à sortir, voilà déjà un moment qu'elles séjournent dans le hall où elles regardent une vitrine d'objets exposés à l'origine de l'émotion qui déclenche le mouvement et même le revirement de la sortie mécanique à l'effraction du musée. Cet instant précis, la narratrice le relate de son côté. Le corps de l'autre ne donne pas signe du désir de partir car l'émotion ressentie devant les vitrines n'a pas été pleinement assouvie (§1).

Mais dans la chronologie des faits, c'est l'excès d'émotion de la narratrice, alors qu'elle lit la lettre d'un soldat disparu, qui suscite chez la protagoniste une première réaction de défense : ceux qui n'ont pas vécu cela sont bien trop sensibles (§ 13), puis une autre, d'empathie, par rapport au mari qui a étouffé dans le poisson, qui l'amène à se nicher dans le ventre de la bête (§§ 10-11, 14-15). De la sorte, la souffrance se communique d'un corps à l'autre. C'est après avoir vu son accompagnatrice essuyer des larmes, à contretemps (§ 13), que la protagoniste réagit. 
De fait, c'est l'émotion de la narratrice devant la vitrine (\$2) qui induit une réaction en chaîne. Telle Emma Bovary avec son parapluie dans son jardin, elle remue avec ses deux parapluies les feuilles mortes d'automne, elle remue le passé en somme, et ce contact physique et sensori-moteur la ramène, par association avec les feuilles séchées vues au musée, à remuer la douleur de l'histoire. Ces feuilles expriment en effet, par métonymie, les corps et les vies de celles qui ont accompli de leurs propres mains, comme elle plus tard, le geste laborieux de collage quand le cellophane n'existait pas encore. Le geste manuel difficile est revécu. L'origine lointaine de quelques feuilles écarlates - d'un rouge feu, en somme - ramène, toujours par glissement d'image, au lointain foyer de feu qui a causé la mort de ces jeunes filles et aurait pu aussi bien, à quelques années près, causer celle de la narratrice, qui ressent dans son corps qu'elle a survécu - contingence de l'Histoire.

Mais la narratrice est aussi sensible aux objets brutaux et cruels laissés par les hommes, et c'est une autre violence qui se communique à elle. Elle se montre plus tard en train de lire la lettre poignante d'un kamikaze, d'autant plus douloureuse que celui-ci dit qu'il n'a pas vécu, pas assez pour vivre de banales expériences (dettes, femme, voir annexe), aspect poignant des lettres de tokkôtai.in (§ 13). Dans l'une d'elles, la mention de l'absence de femme, particulièrement émouvante, annonce l'absence de communication entre la femme et le mari, et fait passer une émotion. L'époux écrit plus qu'il ne parle. Le corps souffre bien assez, à la vue de ces objets qui signalent la souffrance d'autres corps, pour verser des larmes qui vont elles-mêmes nouer l'émotion et mobiliser le corps de la protagoniste. Le sentiment est ici vécu à l'échelle collective, plus côté féminin que masculin, avec une nette opposition entre objets masculins, rudes, et objets féminins pleins de tact - non sans stéréotypes.

Cela dit, de la part de la narratrice, il s'agit là d'une souffrance contrôlée, car elle se retourne très pudiquement pour sangloter et s'attend d'ailleurs ensuite à pareille réaction du côté de son amie $(\S 3)$. La souffrance s'exhibe (larmes, mouchoir), mais sans se dire plus que cela. Elle s'imagine chez l'autre par impression, mais sans que la chose soit verbalisée. Néanmoins, le courant passe entre le corps qui a souffert l'ordalie martiale et le corps vivant sensible. La narratrice est d'autant plus dans la norme sociale qu'elle a le frisson en sentant que son amie veut se faire enfermer et veut violer l'interdit muséal (§3). Elle pose des sens possibles à cet acte, autant de pistes pour le lecteur. 


\section{Le corps souffrant à l'aune de l'Histoire}

Du côté de la protagoniste, le rapport au corps souffrant ne relève pas tout à fait de la convention émotionnelle. Il se traduit à deux niveaux. D'abord, dans l'imagination du corps souffrant du premier mari. Ensuite, par l'insertion du vécu corporel dans une histoire de vie, et même de vie conjugale, qui s'étale sur plusieurs années.

Si la narratrice ressent bien dans son corps la douleur qui lance le récit et mobilise son amie, la trajectoire de celle-ci, bien antérieure, s'inscrit dans une réaction face à l'événement brutal de la disparition du mari. On passe certes d'une mort dite étrange à une autre mort étrange, mais dans une perspective, cette fois, qui engage le rapport de la protagoniste à la société $(\S \S 2,4)$. Le récit exprime la tension, qui n'est pas forcément contradiction, entre le détachement apparent de la veuve, qui se trouve diverses raisons de repousser l'échéance de la visite, et la pensée qui l'obsède de la visite de ce lieu où son mari est vénéré comme un dieu (\$§5-7). Il y a là un refoulement de souffrance, qui tient fondamentalement au déficit de communication avec le premier mari, aggravé de la médiatisation du deuil par le rituel muséal.

Le récit, dans l'optique de la protagoniste, la montre étrangère à cette entreprise militaire. Elle ne sait ni où ni quand son mari décède. Elle en apprend juste assez pour savoir qu'on l'a promu dieu (§ 4). La violence de l'Histoire, la récupération qui s'ensuit, l'aliènent à son époux. Du deuil en tant que tel, le récit ne fait pas état. Néanmoins, il est clair que cet incident a bien contribué à la déposséder encore plus du lien déjà ténu avec son mari en raison du déficit de communication. De même, les sollicitations de l'entourage (§ 5) sont vécues par elle comme une manière de normaliser la frustration en rite funéraire obligé, en visite très orthodoxe du lieu, en génuflexion devant l'Histoire imposée. Non sans irrévérence, la veuve juge que les objets exposés ne sont pas de mise, ce qui constitue une sorte de blasphème à l'encontre de l'embaumement muséal et de l'idéologie martiale (§ 12).

Lorsqu'elle accède enfin à l'espace sépulcral, dans une lumière tamisée à souhait, c'est la sensation d'étouffement de son mari qui la gagne et la pousse à prolonger l'expérience, à se mettre à la place du mari pour ressentir ce qu'il a vécu $(\$ 11)$. Suit une scène de transgression, d'un érotisme pervers, douloureuse et jouissive, masochiste en somme, par lequel la protagoniste reproduit le mouvement pénible du mari dans sa cage de fer, mais que l'on peut lire aussi comme une sorte de commerce sexuel, une tentative pour retrouver une intimité, qui n'aboutit pas (§§ 15-16). Ce que revit dans son corps la protagoniste, c'est bien l'histoire d'une fin horrible issue d'une Histoire violente. L'épopée sacrificielle des tokkôtai est ici suggérée. On a fait 
Corps sensible et corps pratico-inerte : femme frustrée et kamikaze mutique

s'enfermer ces hommes dans ces boîtes pour les envoyer à la mort certaine et nécessaire - hisshi. Le mari, lui, a répondu à l'esprit de corps de l'armée, incarné par le poisson. Le refoulé de l'Histoire affleure pour qui perçoit le non-dit, mais cette lecture-là est compatible avec une autre lecture, qui fait l'économie de l'allusion historique et focalise la relation conjugale. Il s'ensuit que la violence historique, pourtant bien réelle, n'a jamais été que la cause occasionnelle d'un incident révélateur de l'absence de communication entre mari et femme.

\section{Le corps souffrant à l'aune de la relation conjugale}

S'il ne fait aucun doute que l'Histoire a produit cette violence dont l'effet a rejailli sur la vie des couples en les séparant arbitrairement et à jamais, et que la chose est dûment signifiée dans la présence brévissime du mari au foyer conjugal, il n'en reste pas moins que le couple de jeunes gens, ici la protagoniste et son premier mari, communique peu et que la femme ne voit guère ce que son mari attend d'elle - si tant est qu'il attende quelque chose - et cherche à comprendre afin de joindre les deux bouts de sa vie (§ 16). Cet aspect relationnel l'emporte sur la dimension proprement historique du récit.

C'est après avoir vécu l'ordalie du mari de l'intérieur du poisson, mais sans avoir pu communiquer avec lui, même indirectement, que l'épouse, dans une analepse, se souvient que son mari était un homme compliqué, au tempérament difficile. Les paroles de ce mari aussi laconique qu'impératif sont reprises et évaluées, mais non sans ironie. Sa phrase "On doit faire vite » (wareware wa isoganakerebanaranai), son expression apparemment favorite peut s'entendre au sens de l'urgence historique. La guerre est là, il faut profiter d'être ensemble; le vent se lève, il faut tenter de vivre. Il serait aisé de voir ici le drame qui sépare les êtres, et sans doute cette approche est-elle plausible. La veuve y lit un possible appel à un comportement social (faire un enfant ?) dont la nature n'est pas précisée. Mais il se trouve que l'injonction maritale est disqualifiée par l'un de ses contextes d'usage, car elle est prononcée au lit et rendue triviale. À la scène érotique avec le poisson succède alors une autre scène érotique, mais aussi peu concluante que la première, où le mari pressé fait la leçon à sa femme, assise à un coin de lit - ce qui évoque un coït inabouti. C'est parce qu'elle ne savait pas ce que son époux exigeait d'elle que sa femme déploie un transport de tendresse qui n'a pour réponse qu'une phrase castratrice : « Je ne veux pas que tu dises n'importe quoi. » Cet ordre est, pour cette femme, l'acte de frustration qu'elle va refouler des années durant, avant un effort ultime de communication. Si le poisson, animal de chair, est ici de 
fer, c'est que le mari, renfermé et replié sur lui-même, est aussi un homme de fer, hermétique...

C'est vers la fin du récit, dans un processus de remémoration, où le contact physique au sein du poisson alterne avec le souvenir, que se situe cette étape, qui explique l'attitude de la femme à partir du moment où elle a appris la mort de son premier mari. La communication avec lui s'était brisée, elle l'avait perdu comme interlocuteur de chair et de sang (ce n'était plus qu'un dieu pour tout le monde, elle comprise), mais la frustration n'en avait pas moins persisté, cachée par tout un dispositif de rationalisation (elle s'en fichait, elle craignait l'obscurité du lieu), jusqu' au moment où elle avait décidé de prendre l'initiative et d'aller se réapproprier son mari. Le corps souffrant réagit et résiste et à la frustration conjugale et à l'emprise idéologique de la société. L'expérience indirecte avec le mari se solde, malgré la ferveur érotique de l'épouse, par une absence de réponse à ses questions. Par-delà la mort, la relation n'est pas rétablie. Il a fallu toute une trajectoire pour que l'épouse reprenne la main, soit sensibilisée à l'expérience martiale du mari et tente de refaire corps (ittai) avec lui. Mais jamais elle ne saura si son mari a vraiment tenu à quitter sa cage sous-marine : sortir, c'est-à-dire la rejoindre. Le manque de communication renvoie à la faillite du corps conjugal, que l'Histoire n'a fait qu'aggraver pour des raisons qui restent à éclaircir.

Après ce fiasco apparent, la protagoniste s'éloigne un peu du poisson, reprend de la distance avec lui, pour réévaluer la situation (\$18). S'étalant sur plus d'un demi-siècle, le récit fait apparaître le devenir conjugal d'une femme, de la femme peut-être, à travers la guerre et surtout l'après-guerre, dans le passage d'un mari à l'autre. L'enjeu est de continuer la vie présente sans se fixer sur un passé frustrant. L'épouse le fait en jouant de l'interaction entre ces deux maris dont il semble bien que la parole exerce un poids considérable sur son être-au-monde. En l'occurrence, c'est la phrase du second mari qui a un effet libérateur. Dire à sa femme que le premier mari était en partance pour la guerre, c'est vouer ce dernier à la guerre, à la mort certaine, donc le ranger du côté de l'Histoire, et non plus de son épouse. Le verbe utilisé, iku, très fréquent dans les lettres de kamikaze (voir annexe), signifie aller au sens de partir à la guerre, et, dans ce cas précis des tokkôtai, partir à la mort. Le propos du second mari ne fait pas que d'énoncer le déterminisme de l'Histoire - le premier mari était voué à mourir -, il implique que celui-ci a fort bien pu opter pour le trépas (§ 19). Quoi qu'il en soit, l'épouse frustrée saisit mieux le sens de ce qui lui est arrivé. Dès lors, elle a une chance de passer de l'ombre à la lumière et de vivre mieux. Peut-être bien, suggère le récit, que quelqu'un lui a transmis ce désir, que son mari s'est bien manifesté de quelque façon, en lui faisant comprendre 
Corps sensible et corps pratico-inerte : femme frustrée et kamikaze mutique

quelque chose de sa vie, quelque chose qu'elle pourra communiquer à son tour $(\S 20)$.

\section{Le corps souffrant comme manque à dire et la littérature}

L'expérience du poisson, qui trouve son origine dans une conjugalité empêchée, atteste la fonction salutaire de la souffrance que s'impose le corps féminin, douleur certes, mais mâtinée de jouissance, pour négocier la communication avec le corps masculin. L'itinéraire de l'épouse illustre la difficulté, voire l'impossibilité de dire, source d'une frustration conjugale qui ne trouve de dénouement que bien plus tard, au terme d'une dialectique entre souffrance silencieuse, cachée derrière toutes sortes de bonnes raisons, et aspiration à vivre et à retrouver une continuité existentielle. La résurrection intégrale du passé à la lumière du passage doux-amer permet en quelque sorte de combler le hiatus historico-sentimental qui grève la vie de l'épouse.

Mais il reste à comprendre pourquoi elle ne raconte pas cela elle-même, pourquoi sa parole est déléguée à une autre qu'elle-même, certes expressément solidaire en tant que femme, mais qui n'est pas elle. Face à ce qu'elle vit, l'épouse reste interdite. Non contente d'écouter la parole d'autrui, de ce premier mari dont elle ne saisit pas les exigences, de ce second mari dont les paroles ont pu la tromper, elle subit sans mot dire la parole de la société sur ce qu'elle doit faire, et elle ne trouve pas les mots en découvrant la fin horrible de son mari. Il ne faut sûrement pas en conclure au silence imposé à la femme sous diverses formes (elle colle des fleurs séchées, l'homme écrit des lettres) en régime homo-sociétal, mais il est clair que quelqu'un doit dire à sa place ce qu'elle ne peut pas dire.

Là, l'Histoire échoue, laisse entendre la nouvelle, car elle subsume la souffrance individuelle sous la grande guerre nationale et ravit le corps des soldats aux proches de ceux-ci. À la date de 1976, il n'est que de constater que l'historiographie, même si s'ouvre le champ des mentalités, n'approche guère le vécu individuel, et moins encore celui des femmes. Le sanctuaire Yasukuni, certes visitable par tous, reste un espace masculin. En revanche, c'est à la littérature, en tant qu'elle fait preuve d'imagination - c'est le cas de la narratrice -, de recréer, de restituer, de recomposer ce que l'historien peine encore à dire et surtout ce que le sujet féminin de l'Histoire achoppe à formuler. Dans Requiem (1935-1940) d'Anna Akhmatova (1889-1966), une femme demande à la poétesse, qu'elle vient de reconnaître dans la foule des épouses et des mères qui attendent devant la prison de Moscou d'avoir des nouvelles de leurs maris et de leurs fils emprisonnés : «Et ça, vous pouvez le 
décrire ? », et Anna répond qu'elle le peut, oui ${ }^{3}$. La littérature peut, en effet, restituer ce corps souffrant qui ne se dit pas, et restaurer le rapport de la femme aussi bien à l'Histoire, dont elle a été exclue par les instances masculines, qu'à son vécu conjugal personnel, brimé lui aussi par la censure verbale de l'homme. Kôno Taeko va plus loin, avec ce poisson fantastique en fer, qui concentre le drame historique du Japon à travers le regard féminin, qui dit le corps souffrant du Japon à travers la condition féminine, mais qui amorce aussi un discours subversif de libération.

Avant de conclure, il convient de replacer cette nouvelle dans l'histoire de la littérature écrite par des femmes au Japon. Une bonne partie de celle-ci ne peut se comprendre si l'on ne tient compte du clivage social entre les mondes masculin et féminin. L'homme est censé s'épanouir au travail, la femme au foyer et dans la maternité, et cette distribution des rôles, cette division du travail, passe pour consensuelle. Décalée par rapport à la norme masculine, la femme est l'être à part et qu'il faut protéger. Telle est la doxa. S'il arrive bien que la femme réagisse et résiste, la femme japonaise reste le plus souvent inconsciente de cette situation qui peut la desservir ou s'identifie au sexe dit fort dans son comportement. Telle est la tendance. Les mythes et préjugés sur la nature de la femme circulent, qui contribuent à pérenniser la séparation des univers masculin et féminin et sont à imputer au substrat confucéen, néoconfucéen et bouddhique.

Cette dichotomie socio-sexuelle caractérise la littérature japonaise contemporaine : les auteurs masculins y écriraient de la littérature, et les auteurs féminins, de la littérature pour femmes. Cette distinction engendre tout un cortège de stéréotypes, notamment celui selon lequel la sensibilité féminine fonctionnerait sur le mode émotionnel, a- ou illogique et pointilliste. En fait, au moins depuis le début du $\mathrm{XX}^{\mathrm{e}}$ siècle, la Japonaise qui écrit a établi sa position professionnelle d'écrivain et atteint un haut niveau d'indépendance économique. Il n'en reste pas moins qu'elle a dû lutter contre sa relégation systématique dans la littérature féminine. Et si le corps constitue l'un des thèmes et motifs saillants de cette littérature féminine japonaise, c'est qu'il est l'arme absolue de défense, d'affirmation et d'illustration contre un monde masculin impénétrable.

La nouvelle de Kôno Taeko rompt avec les représentations masculines de la guerre en lui opposant la transgression massive, contre-nature, d'un corps qui surmonte le fossé de l'Histoire au masculin en retrouvant le lien rompu avec le corps de l'autre, de l'homme. Cette expérience de la guerre, étonnante, incompréhensible, douloureuse au premier chef, est exorcisée par une

\section{Akhmatova 2007.}


Corps sensible et corps pratico-inerte : femme frustrée et kamikaze mutique

copulation symbolique, passablement scandaleuse, avec la cause du mal, la guerre littéralement incarnée dans le poisson, un poisson reconstitué pour tous les besoins de la muséographie et de l'exemple militaro-religieux, un poisson qui a fait exploser et s'éparpiller aux quatre coins de la mer le corps du mari, en une sorte de ragoût pour poissons. C'est, en définitive, une belle épreuve de résistance du corps souffrant féminin à l'emprise de l'Histoire masculine que donne à lire la nouvelle de Kôno Taeko, mais aussi d'une littérature au féminin qui resémantise les oripeaux martiaux masculins au profit d'une relation féminine restaurée avec l'existence. 


\section{Gérard Siary}

\section{ANNEXE \\ LETTRES DE KAMIKAZES}

Nota bene - Ces traductions, à la différence des traductions françaises déjà en circulation mais retraduites de l'anglais, parfois très librement ou peu soignées, sont établies à partir de l'original.

\section{UEHARA Ryôji}

Depuis vingt ans que j'ai reçu la vie, j'ai joui d'une éducation sans aucun inconfort, j'ai été heureux. Dans la douce chaleur de votre amour, mes chers parents, et de par la diligence de mon grand frère et de ma petite sæur, les meilleurs du monde, j'ai pu couler des jours agréables. Et puis, ne serait-ce qu'un peu, j'ai parfois été égoïste. Entre-temps, de tous vos enfants, celui qui vous a causé le plus de souci, mes chers parents, c'est moi. De partir avant vous, sans vous rendre tous vos bienfaits, cela me fend le cour, mais au Japon où loyauté au Tennô et piété filiale ne font qu'un, et où se donner tout entier au Tennô, c'est pratiquer la piété filiale, je crois que vous voudrez. bien pardonner mon geste.

Membre des forces de l'air, je mène une vie où chaque jour a la mort pour prémisse. Chaque lettre que je trace, chaque mot que je dis, auront été mes derniers. Tout làhaut dans le ciel, jamais on ne focalise sur la peur. Se peut-il que je meure ainsi, par percussion? Mais non, de toute façon, cette pensée de la mort ne me vient pas. Et d'ailleurs, il m'est arrivé d'éprouver le besoin pressant de percuter ainsi. La mort ne me fait aucunement peur. Je ressens plutôt de la joie. Car je crois, quoi qu'il arrive, que je pourrai retrouver mon frère aîné Rŷ̂. Et justement, nous revoir dans les cieux, c'est là mon vou le plus cher. Quant à ce qu'on appelle la conception de la vie et de la mort, je n'en ai pas eue. Parce que, de toute façon, je me suis dit que cette conception de la vie et de la mort, ce désir de donner absolument sens et valeur à la mort émanait d'une peur obscure de la mort. Moi, comme je crois que les retrouvailles aux cieux passent par la mort, je ne puis avoir peur d'elle. La mort, quand je songe qu'elle est le processus pour monter aux cieux, n'existe point.

Plus précisément, j'ai aspiré au libéralisme. Car àmon sens, pour que le Japon continue vraiment et pour toujours, le libéralisme était une nécessité. Il vous semble entendre des idioties. C'est parce qu'actuellement, le Japon est plongé dans le totalitarisme. Et pourtant, si j'ouvre bien les yeux, et que je réfléchis à la nature réelle de l'être humain, le libéralisme me paraît justement une doctrine rationnelle.

Si l'on regarde les victoires et les défaites à la guerre, si l'on regarde la doctrine du pays, on a confirmation avant terme. La victoire du pays qui possède une doctrine naturelle et conforme à la nature de l'être humain est plus évidente que de voir le feu brûler. 
Mon idéal d'un Japon pareil au grand empire britannique de jadis s'est évanoui, vaincu. Mais au moins pour la liberté et l'indépendance du Japon, je suis heureux de sacrifier ma vie. [...]

Dans le tiroir droit de ma bibliothèque qui est dans l'annexe, il y a un livre que je vous laisse en souvenir. Si le tiroir ne s'ouvre pas, ouvrez le tiroir de gauche et sortez le clou. Voilà, je forme des voux pour que vous preniez bien soin de vous.

Mes meilleurs sentiments à mon grand frère, à ma sœur Kiyoko et aux autres.

Au revoir et bonne santé.

Adieu pour toujours.

À mes parents

$R y \hat{o j i} i^{4}$

\section{WATANABE Kôzô}

Chers parents, comment vous portez-vous depuis la dernière fois?

Voilà vingt années maintenant que Kôzô a reçu la vie en ce monde et a été élevé sans souci ni peine par des parents affectueux. Durant tout ce temps, ma santé étant plus fragile que mes frères, je vous ai toujours causé des inquiétudes, mes chers parents. Un jour, alors que j'étais longtemps alité, tout près de mourir, et puis toutes les fois où j'ai gardé le lit, vous m'avez veillé sans prendre de sommeil : je vous en sais gré aujourd'hui. Néanmoins, je regrette d'avoir passé tous ces jours sans avoir marqué la moindre piété filiale.

L'édit impérial de mobilisation générale a été proclamé le 8 décembre 1944, et la situation s'aggrave de jour en jour. Comment mon jeune cour pourrait-il ne pas réagir? Vu la situation actuelle, j'ai pensé que c'était manifester ma piété filiale au mieux et j'ai quitté la maison.

Depuis lors, je suis dans l'aéronavale. Chacun de mes vols peut être le dernier et, votre photo sur mon cæeur, je poursuis mon entraînement intensif. Et puis, comme vous le savez, chers parents, les aigles divins, nos compagnons des unités spéciales d'attaque, sont apparus à cette heure des plus critiques pour l'Empire. Comment Kôzô, qui tient de votre sang, pourrait-il ne pas les suivre? Un homme peut-il avoir d'autre satisfaction que celle-là? De même, pour vous mes parents, je pense que c'est bien le moindre des actes de piété filiale, et je perfectionne ma technique tous les jours en rêvant de voir arriver le plus vite possible le jour de la sortie.

Quand je prendrai mon envol, comme il est évident que de toute façon je tomberai sous le tir d'un navire ennemi ou un avion ennemi, et que mon corps ne sera pas rendu, j'inclus au moins, pour que vous gardiez souvenir de Kôzô, quelques cheveux.

Quand j'y pense, cher père, ce que je déplore le plus, c'est au fond de vous voir vous affaiblir.

4. Chiran kôjo nadeshiko-kai 1996 : 76-78. 


\section{Gérard Siary}

Veuillez me pardonner, chers parents, de vous précéder dans le trépas.

Depuis mon départ, chers parents, je vous crois préparés à la situation présente, mais je vous souhaite de tout cour de ne surtout pas vous décourager et de vivre une très longue vie.

Grand frère Kiyoshi,

Grand frère Motomasa,

Grande sœur Fujiko, grande-sœur Hanai,

Kimie, Kinoi, mes petites sœurs chéries, je vous souhaite à toutes de vivre sans problème. Je vous confie juste de manifester à nos chers parents, en mon lieu et place, votre piété filiale.

- Je n'ai contracté ni prêt ni emprunt.

- Je n'ai eu aucune relation avec des femmes.

À mes chers parents

$K \hat{o} z \hat{o}^{5}$

5. Chiran kôjo nadeshiko-kai 1996 : 124-125. 
Corps sensible et corps pratico-inerte : femme frustrée et kamikaze mutique

\section{BIBLIOGRAPHIE}

Akhmatova, Anna (2007). Le Roseau. Traduit du russe [Trostnik] par Mouze, Christian. Corbières, Harpo \&, non paginé.

AXELL, Albert, KASE, Hideaki (2002). Kamikaze. Japan's Suicide Gods. New York, Pearson Education Ltd.

CHIRAN KÔJO NADESHIKO-KAI 知覽高女なでしこ会 (dir.) (1996). Gunjô -Chiran tokkôkichi yori 群青一知覧特攻基地より. Kagoshima, Taki shobô.

DIX, Monika (2009). «Saint or Serpent ? Engendering the Female Body in Medieval Japanese Buddhist Narratives ». In TURNER, Bryan S. et ZHENG, Yangwen (dir.) (2009). The Body in Asia. Oxford/New York, Berghahn Books.

DONATH, Diana (2012). Frauenthematik in der japanischen Literatur. Zwanzig Autorinnen der 1960er bis 1980er Jahre. Munich, Iudicium : 147-150.

HENRY, Michel (2000). Incarnation. Une philosophie de la chair. Paris, Seuil.

KÔNO, Taeko (1989). «Iron Fish ». In GeSSEL, Van C. et MATsumOTO, Tomome (dir.), The Shôwa Anthology. Modern Japanese Short Stories, tr. Yukiko Tanaka. Tokyo/ New York, Kodansha International : 363-374.

KôNO, Taeko 河野多惠子 (1995). 《Tetsu no uo » 鉄の魚. In Kôno Taeko zenshû 河野多 惠子全集, vol. 4. Tokyo, Shinchôsha : 39-49.

KôNo, Taeko (1996). « Der Eisenfisch ». In Taeko Kono : Knabenjagd, Trad. et dir. HIJIYA-KIRSCHNEREIT, Irmela. Francfort, Suhrkamp : 123-139.

Langton, Nina Jean (1993). On the Edge: Woman, Nature, Mother and Father in the Works of Kôno Taeko. Thèse inédite, The University of British Columbia.

MORI Shirô 森史朗 (2006). Tokkô to wa nanika 特攻とは何か. Tokyo, Iwanami shoten.

LEBRA, Takie Sugiyama (1984). Japanese Women. Constraint and Fulfillment. Hawaii, Hawaii University Press.

Morley, Patricia (1999). The Mountain Is Moving. Japanese Women's Lives. Vancouver, UBC Press : 134-135.

NishitANI, Osamu (2013). « Corps ». In LegENDRE, Pierre (dir.), Tour du monde des concepts. Paris, Fayard, Kindle : 4633-4652.

RECORD IN PiCtURES OF YÛSHÛKAN(2009). Tokyo, Yasukuni Shrine.

TAKAHASHI, Tetsuya 高橋哲哉 (2005). Yasukuni Mondai 靖国問題 (Morts pour l'empereur. La question de Yasukuni). Tokyo, Chikuma shobô. Trad. fr. NANTA, A. Paris, Les Belles Lettres, 2012. 
Gérard Siary

\section{GLOSSAIRE}

Dainihon Hokkekyô genki 大日本法華経験記

Dôjoji engi emaki 道成寺縁起絵巻

gunshin 軍神

hisshi 必死

$i k u$ 征<

ittai 一体

kaiten 回天

kamikaze 神風

Kôno Taeko 河野多惠子

setsuwa 説話

tokkôtai 特攻隊

Yasukuni jinja 靖国神社

Yûshûkan 遊就館

wareware wa isoganakerebanaranai 我々は急がなければならない 